\title{
Controlling nanoparticle synthesis derived from bimetallic metal-organic frameworks
}

\author{
Zhihengyu Chen ${ }^{1}$, Zhijie Chen², Omar K. Farha ${ }^{2,3}$, Karena W. Chapman ${ }^{1}$ \\ ${ }^{1}$ Department of Chemistry, Stony Brook University, Stony Brook, New York 11794, United States, \\ ${ }^{2}$ Department of Chemistry and International Institute for Nanotechnology, Northwestern University, \\ Evanston, Illinois 60208, United States, \\ ${ }^{3}$ Department of Chemical and Biological Engineering, Northwestern University, Evanston, Illinois 60208, United States
}

karena.chapman@stonybrook.edu

Metal-organic frameworks (MOFs) have been recently known as novel precursors in nanomaterial synthesis. To understand the mechanism behind the phase transformation in atomic scale, we apply in-situ X-ray pair-distribution analysis to monitor the whole process, from distortion, destabilization, partial reduction, to the eventual nanoparticle formation and defect evolution of a series of bimetallic MOFs PCN-250. These MOFs with different trimeric node composition $\left(\mathrm{Fe}_{3}, \mathrm{Fe}_{2} \mathrm{Co}\right.$, and $\left.\mathrm{Fe}_{2} \mathrm{Ni}\right)$ allow us to control the structure, chemistry, and defect of resulting nanoparticles. Notably, we found selective reduction of Ni from the node with defect-rich frameworks retained. This can be a new route for future MOFs crystal engineering.

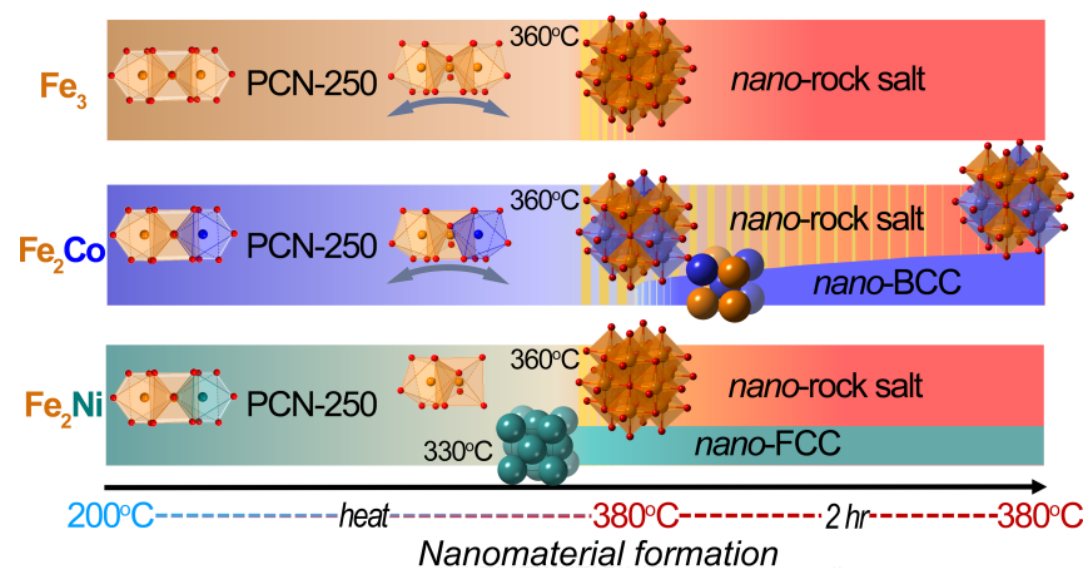

Figure 1. The structure of MOFs and resulting nanophases are tracked through PDF analysis.

[1] Chen, Z., Chen, Z., Farha O. K. \& Chapman, K. C. (2021). J. Am. Chem. Soc. 143, 8976.

\section{Keywords: Pair-distribution function; nanomaterial synthesis; metal-organic frameworks; solid solution}

Any This work was supported as part of the Inorganometallic Catalyst Design Center, an Energy Frontier Research Center funded by the U.S. Department of Energy (DOE), Office of Science, Basic Energy Sciences (BES), under Award DE-SC0012702. This research used resources of the Advanced Photon Source, a U.S. Department of Energy (DOE) Office of Science User Facility operated for the DOE Office of Science by Argonne National Laboratory under contract no. DE-AC02-06CH11357. 